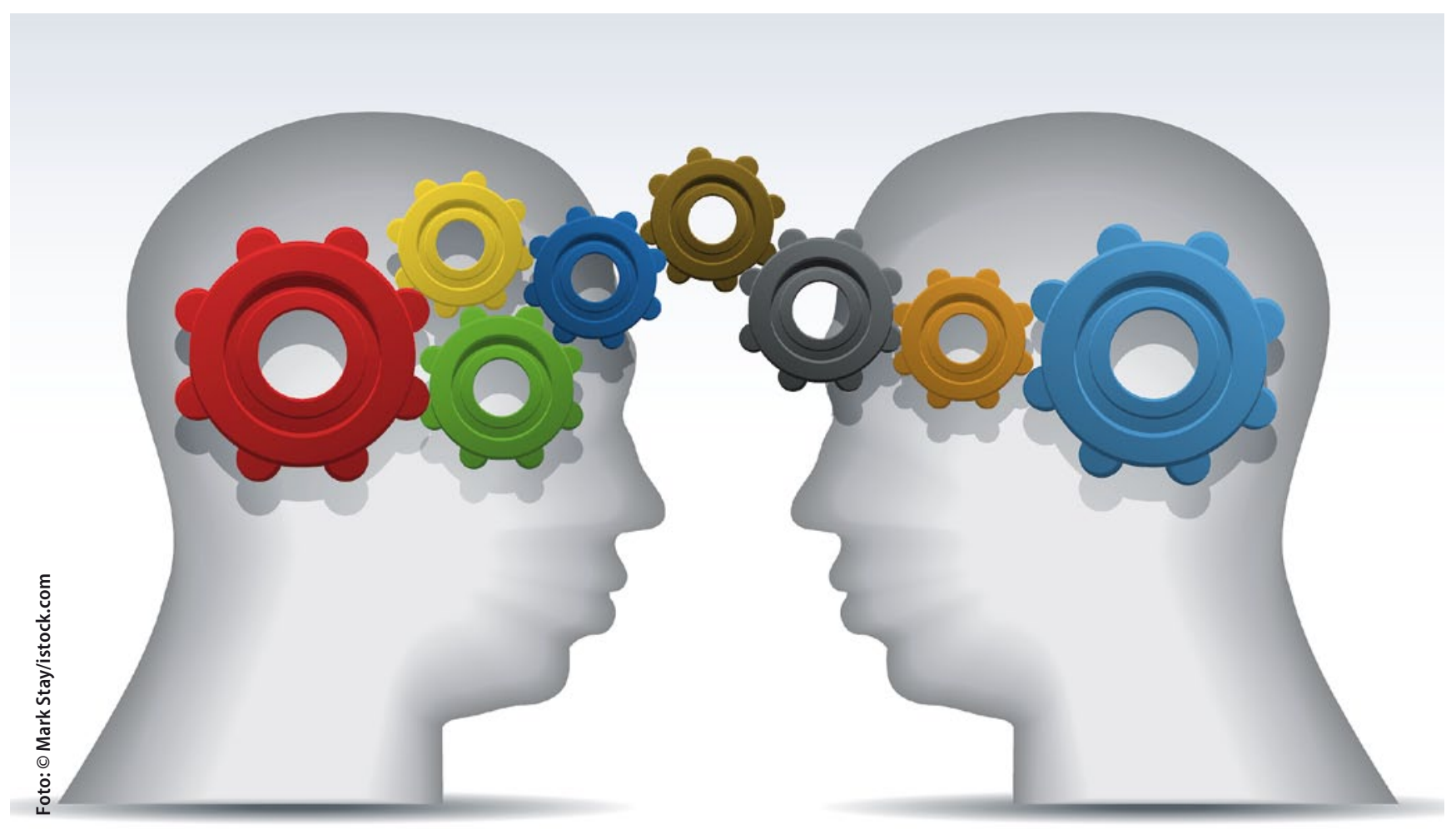

\title{
Zwölf Mythen der Inhouse-Weiterbildung im Marketing
}

Inhouse-Weiterbildungen sind für viele Unternehmen seit Jahren ein fester Bestandteil der Personalentwicklung ihrer Marketeers. Dennoch bestehen falsche Vorstellungen darüber, was erfolgreiche innerbetriebliche Weiterbildungsprogramme auszeichnet. Der Beitrag beschreibt zwölf zentrale Mythen in der Inhouse-Weiterbildung im Marketing, stellt diese der Realität gegenüber und skizziert Handlungsempfehlungen für Unternehmen.

CHRISTIAN BELZ | MICHAEL BETZ | PHILINE WERNER

nhouse-Weiterbildungsprogramme sind en vogue (Löhmer 2012, S. 21). Eine Studie von McKinsey belegt beispielsweise, dass Unternehmen jährlich weltweit 100 Milliarden US-Dollar für die Weiterbildung ihrer Mitarbeiter ausgeben (DeSmet/ McGurk/Schwartz 2010). Dennoch ist vielen Unternehmen nicht klar, wie maßgeschneiderte Programme idealerweise zu konzipieren, zu planen und durchzuführen sind, so dass sich ihre Erwartungen letztlich auch erfüllen. Dies gilt insbesondere für die Ausund Weiterbildung von Fach- und Führungskräften im Marketing, deren Arbeitsalltag durch neue und immer spezifischere Themen geprägt ist. Marketeers werden neue Fähigkeiten abverlangt, über die sie jedoch zumeist nicht verfügen (Gök/Hacioglu 2010, S. 300; Wyner 2008).
Der vorliegende Beitrag stützt sich auf die branchenübergreifende praktische Erfahrung der Autoren, die als externe Trainer und Berater in über 60 großen mittelständischen Unternehmen und börsennotierten multinationalen Konzernen (kleine und Kleinstunternehmen werden nicht berücksichtigt) erfolgreich Inhouse-Weiterbildungsprojekte im Marketing durchgeführt haben. Betrachtet werden sowohl Projekte aus dem Konsumgüterbereich (wie z. B. FMCG, Telekommunikation oder Banken und Versicherungen) als auch aus dem Business-to-Business-Umfeld (wie z. B. Maschinen- und Anlagenbau, Medizinalgüter, Pharma oder IT). Gerade im Business-to-Business-Marketing spielt der Vertrieb eine zentrale Rolle und kann im Rahmen des MarketingMix der Distributionspolitik zugeordnet werden. Aus diesem 
Grund schließen die untersuchten Programme inhaltlich neben den Themen

- Marketingstrategie,

- operativer Marketing-Mix,

- Marketinginnovation,

- Markenführung,

- internationales Marketing,

- Marketing-Performance oder

- Multi-Channel-Management auch die Themen

- Sales Management bzw.

- Smart-, Key- und Global-Account-Management explizit mit ein. Die methodische Ausgestaltung reicht von Impulstrainings über Entwicklungsworkshops mit Experten-Input bis hin zu mehrmoduligen interaktiven Schulungskonzepten mit Transferphasen. Aus dieser Erfahrung heraus lässt sich ein generischer Prozess für Inhouse-Weiterbildungen mit den Teilschritten „Identifikation Weiterbildungsbedarf“, „Auswahlprozess Weiterbildungspartner“, „Konzeption“, „Durchführung“, „Transfer“ und "Multiplikation“ ableiten. Des Weiteren lassen sich zu den einzelnen Phasen diverse Mythen identifizieren (vgl. Abbildung 1). Diese Mythen werden der Realität in der Praxis gegenübergestellt und mit konkreten Beispielen illustriert. Abschließend werden für jeden Mythos Empfehlungen abgeleitet, die Unternehmen für eine erfolgreiche Realisierung von Inhouse-Weiterbildungen im Marketing beherzigen sollten. Der Erfolg ergibt sich dabei nicht aus positiven Teilnehmer-Feedbacks zu Trainern bzw. zum Training selbst (abgefragt in sogenannten „HappySheets"), sondern äußert sich vielmehr durch veränderte und verbesserte Verhaltensweisen und Prozesse im Tagesgeschäft der Marketeers. Sie manifestieren sich in der Regel innerhalb von drei Monaten nach der Weiterbildungsmaßnahme. Insgesamt konzentriert sich der Beitrag durch die kurze und prägnante Schilderung jedes einzelnen Mythos bewusst darauf, Weiterbildungsverantwortliche in Unternehmen entlang des gesamten Inhouse-Weiterbildungsprozesses zu sensibilisieren. Er liefert somit erste Denkanstöße, anstatt in einzelnen Phasen in die Tiefe zu gehen. Eine solche punktuelle Vertiefung ist ohnehin nur situativ sinnvoll und sollte projektbezogen und fallspezifisch vorgenommen werden.

\section{Mythos 1 -}

\section{Im Marketing arbeiten nur Marketingexperten}

\section{Realität}

Mit Ausnahme von FMCG-Unternehmen wie Procter \& Gamble oder Unilever verfügen Fach- und Führungskräfte im Marketing häufig über keine klassische Marketingausbildung. Vielmehr liegt ihre Grundausbildung nahe an der Leistungskompetenz des jeweiligen Unternehmens. So finden sich beispielsweise in Marketingabteilungen von Medizinalgüter- und Pharmaunternehmen ausgebildete Krankenschwestern, Chemiker, Biologen oder Mediziner; im Maschinen- und Anlagenbau oder in der Telekommunikationsbranche Ingenieure, Physiker und Techniker.

\section{Empfehlung}

Den unterschiedlichen Ausbildungshintergründen und Leistungsniveaus muss in der Inhouse-Weiterbildung, beispielsweise bei der Konzeption und der Auswahl geeigneter Lehr- und Lernmethoden, adäquat Rechnung getragen werden. Es ist wichtig, Trainingsgruppen hinsichtlich ihres Leistungsniveaus möglichst homogen zusammenzustellen und diese z. B. mit gezielten Modulkombinationen zu bedienen. Stellenweise kann es auch sinnvoll sein, die interne Weiterbildung mit qualifizierten überbetrieblichen Programmen zu flankieren, um individuelle Bedürfnisse und Defizite abzudecken.

\section{Mythos 2 - Universitäten und Fachhochschulen bereiten Marketeers auf die Praxis vor}

\section{Realität}

Wenn Unternehmen Absolventen von Universitäten und Fachhochschulen für das Marketing rekrutieren, sind sie oftmals von deren Fähigkeiten enttäuscht, da die akademische Ausbildung oft sehr breit, theoretisch und damit zu wenig anwendungsorientiert ausgestaltet ist. Unternehmensspezifische Konstellationen und konkrete Aufgaben der Verantwortlichen können in diesem Rahmen nicht berücksichtigt werden. Folglich sind Unternehmen gezwungen, den Absolventen ihr unternehmensspezifisches, häufig erfahrungsbasiertes Marketingverständnis und Know-how zu vermitteln, damit diese das operative Tagesgeschäft bewältigen können - ganz gleich ob der Einstieg direkt oder über ein TraineeProgramm erfolgt. Akademisches Wissen wird dabei ausgeblendet, eine Verzahnung beider Welten findet nicht statt.

\section{Empfehlung}

Um eine Brücke zwischen beiden Welten zu schlagen und die jeweiligen Vorteile (Systematik und Methodik sowie Pragmatismus und Erfahrung) nutzbar zu machen, ist es Aufgabe von Inhouse-Weiterbildungsprogrammen, akademisches Wissen aufzufrischen und so zu vermitteln, dass es im Tagesgeschäft anwendbar und umsetzbar ist. Hierbei ist es oft besonders wichtig, dass der externe Trainer sich hinsichtlich Sprache, Methoden und Konzepten an das Unternehmen anlehnt und keine beliebigen und verwirrenden Begriffe und Strukturen einbringt. Nur so kann eine Weiterbildung die Teilnehmenden befähigen, ihre eigenen Initiativen und Lösungsideen professionell zu realisieren.

\section{Mythos 3 -}

\section{Unternehmen entwickeln Marketeers systematisch}

\section{Realität}

In einigen Unternehmen dominiert nicht das Marketing, sondern Vertrieb, Produktentwicklung oder Produktmanagement. Hier ist das Marketing Erfüllungsgehilfe und es sind keine Personalentwicklungspläne oder vordefinierten Karrierepfade für die Marketingmitarbeiter vorhanden. Dies hat zur Folge, dass Trainingsmaßnahmen fach- und mitarbeiterseitig initiiert werden. Gleichzeitig fehlt es an der nötigen finanziellen und zeitlichen Unterstützung sowie an der 
Einbindung der Human-Resources-Abteilung. Das Beispiel eines deutschen Medizinalgüterherstellers illustriert die Konsequenzen einer fehlenden Personalentwicklung: Aufgrund der ungenügenden Kooperation und Koordination zwischen Fachseite und Personalabteilung initiierte die Marketingabteilung aus einem kurzfristigen Aktionismus heraus eigenständig eine Trainingsmaßnahme, die nicht über den Status einer Insellösung hinauskam. Letztlich konnten sich deshalb keine positiven Trainingseffekte einstellen.

\section{Empfehlung}

Vorbild für eine systematische Weiterbildung können einige Konzerne sein, die sich bewusst mit der Fachkarriere im Marketing befassen, damit ihre Mitarbeiter auch anspruchsvolle Marketingaufgaben erfüllen können. Allgemein muss ein ganzheitliches Personalentwicklungsprogramm die Grundlage für Inhouse-Weiterbildung sein. Dieses sollte den individuellen Schulungsbedarf ermitteln, langfristig ausgerichtet sein und sämtliche Weiterbildungsmaßnahmen in ein Gesamtkonzept einbetten. Programme, die einseitig von der Personalabteilung initiiert werden, greifen hier allerdings zu kurz. Vielmehr sind Fachseite, Personalbereich und externer Trainingsanbieter gleichermaßen in der Verantwortung.

\section{Mythos 4 - RfPs und E-Sourcing-Tools helfen bei der Auswahl des richtigen Trainingspartners}

\section{Realität}

Aus Kosten- und Effizienzgründen setzen Unternehmen immer häufiger Ausschreibungen für Inhouse-Weiterbildungsprogramme ein. Dabei greifen sie auf ausführliche Requests for Proposal (RfPs) und E-Sourcing-Tools oder sogar auf die Expertise von unabhängigen Beratern zurück. Diese Instrumente verursachen selbst viel Aufwand und können sogar die Identifikation des richtigen Partners verhindern. Sicherlich haben diese Tools beim Einkauf von standardisierten Produkten ihre Berechtigung. Für die Beschaffung einer maßgeschneiderten Dienstleistung, bei der es auf Persönlichkeit, Sympathie, Vertrauen, gemeinsame Lösungsentwicklung und langfristige Partnerschaft ankommt, sind sie jedoch vollkommen ungeeignet. Darüber hinaus scheuen besonders die besten Inhouse-Anbieter den Aufwand, ihre Mehrwertleistung in einen vorgefertigten Rahmen zu pressen. Denn damit wird ihre Differenzierung zu Wettbewerbern erschwert und preisorientierte Anbieter werden tendenziell begünstigt.

Ein Beispiel für eine derartige Ausschreibungspolitik liefert ein global tätiger amerikanischer IT-Konzern, der während des gesamten Ausschreibungsprozesses außer einem E-Sourcing-Tool, dass von einem strategischen Einkäufer in Rumänien betreut wurde, keine persönliche Interaktion mit dem Fach- und Personalbereich zuließ.

\section{Empfehlung}

Um Anbieter zu finden, die in der Lage sind, bedürfnisgerechte Weiterbildungsprogramme anzubieten und die an langfristigen Partnerschaften interessiert sind, gilt es auf Unternehmensseite den Teilnahmeaufwand an einem RfP so gering wie möglich zu halten, neuen kreativen Lösungsansätzen Raum zu geben sowie den persönlichen Kontakt zu suchen und zuzulassen. So sind Gespräche mit Referenzkunden des Anbieters deutlich aussage-

\section{Abb. 1 Zwölf Mythen der Inhouse-Weiterbildung entlang des Weiterbildungsprozesses}

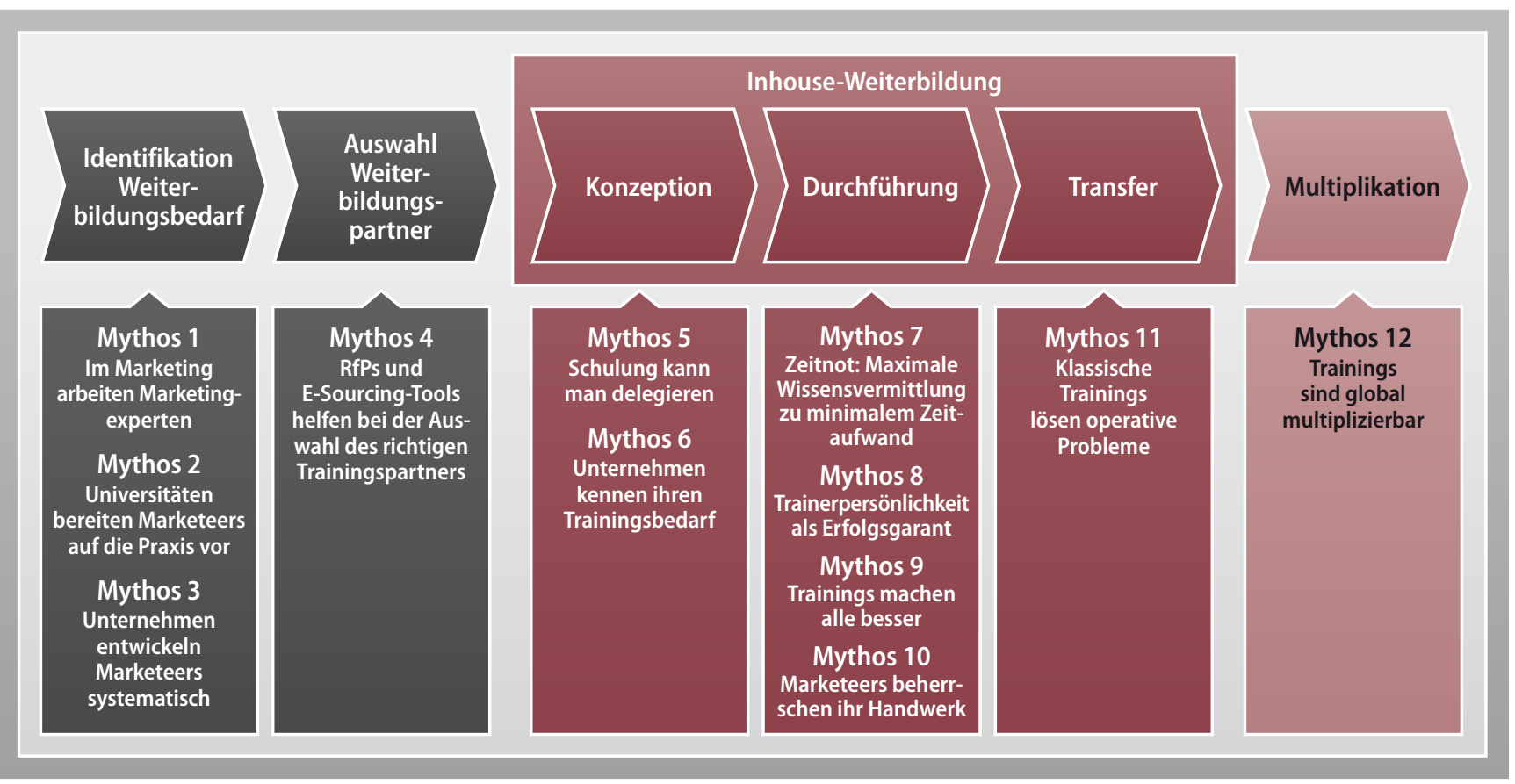


kräftiger als ein E-Sourcing-Tool, in das Informationen eingegeben sind. Nach einer derartigen Vorevaluation lässt sich anschließend der Aufwand von Anbieter und Kunde besser auf die gemeinsame Lösung konzentrieren.

\section{Mythos 5 - Schulung kann man delegieren}

Realität

Das Beispiel eines großen deutschen FMCG-Herstellers verdeutlicht die Konsumhaltung, die bei vielen Unternehmen hinsichtlich der Inhouse-Weiterbildung vorherrscht: Inhaltliche Konzeption, Durchführung sowie Administrationsaufgaben sind komplett an einen externen Partner ausgelagert. Konsequenzen davon sind fehlendes internes Involvement und Commitment zum Training sowie mangelnde Abstimmung und inhaltliche Weiterentwicklung. Weiterbildungsanbieter sind zwar hinsichtlich Inhalt, Methodik und Didaktik stark. Sie sind jedoch in der Regel keine Branchen- und Unternehmensexperten. Folglich bedarf es auf Unternehmensseite eines Sparring-Partners, damit ein Programm wirklich auf die Besonderheiten eines Unternehmens oder einer Branche angepasst werden kann.

\section{Empfehlung}

Mit der Auswahl eines externen Weiterbildungspartners beginnt für das Unternehmen erst die eigentliche Arbeit. Es gilt einen Projektleiter beziehungsweise ein Projektteam zu definieren, das mit dem externen Partner in allen Projektphasen eng zusammenarbeitet und gemeinsam

- Inhalte,

- unternehmensspezifische Fallstudien,

- unternehmenseigene Best Practices,

- Workshops sowie

- das Rahmenprogramm entwickelt,

sodass das gesamte Konzept bedürfnisgerecht ist und aus einem Guss kommt. Wesentlich ist auch die selektive Einbindung, Teilnahme und Mitwirkung von Schüsselpersonen des Unternehmens an der Durchführung der Weiterbildung.

\section{Mythos 6 - Unternehmen kennen ihren Trainingsbedarf}

Realität:

Häufig wenden sich Unternehmen mit konkreten Vorstellungen über Aufbau und Inhalt einer Weiterbildungsmaßnahme an einen externen Anbieter. Jedoch stellt sich schon bei den ersten persönlichen Kontaktgesprächen oder spätestens in der Konzeptionsphase heraus, dass ein klassisches Trainingsformat die beschriebenen Probleme allein nicht lösen kann. Es fehlt an strukturellen und konzeptionellen Grundlagen, auf denen ein Training aufbauen muss, um nachhaltig wirksam zu sein. So zeigt das Beispiel eines global tätigen Maschinenbaukonzerns, der einen Trainingsbedarf im Key Account Management identifiziert hatte, dass zunächst im Rahmen eines Beratungsprojekts die Entwicklung eines unternehmensspezifischen KAM-Konzepts nötig war, um der Arbeit der Key Account
Manager Struktur und Systematik zu geben. Ohne die notwendigen unternehmensspezifischen Infrastrukturen und Handlungsleitlinien verpuffen Trainings- und Motivationseffekte von Weiterbildungsmaßnahmen sehr schnell und wirken oftmals sogar negativ.

\section{Empfehlung}

Für Unternehmen ist es ratsam, nicht in konkreten Weiterbildungsmethoden zu denken, sondern sich vielmehr der eigentlichen Probleme bewusst zu werden. Nur dann lassen sich gemeinsam mit dem externen Partner geeignete Weiterbildungsformate wählen und entwickeln. Damit kommen neben klassischen Trainings auch individuelle Coachings, Train-the-Trainer-Programme oder Hybridansätze (Kombinationen aus Beratungs- und Trainingsprojekten) infrage. Inhaltlich ist es in Hinblick auf den Trainingsbedarf nützlich, von den neuen Strategien, Zielen und Aufgaben der betroffenen Mitarbeitergruppen auszugehen.

\section{Mythos 7 - Zeitnot: Maximale Wissensvermittlung} mit minimalem Zeitaufwand

Realität

Betriebswirtschaftlichem Effizienzdenken entsprechend möchten Unternehmen für die getätigten Weiterbildungsinvestitionen einen „maximalen“ Ertrag erhalten und in der zur Verfügung stehenden Trainingszeit so viel Wissen und Know-how wie möglich vermitteln lassen. Dabei wird jedoch die menschliche Komponente völlig außer Acht gelassen bzw. die Aufnahmefähigkeit der Teilnehmer maßlos überschätzt. Das veranschaulicht das Beispiel eines österreichischen Pharmaunternehmens: Entgegen den Empfehlungen des Trainers bestand die Geschäftsleitung darauf, die Trainingstage eines dreitägigen Trainings jeweils von $8.30 \mathrm{Uhr}$ bis 18.30 Uhr voll auszunutzen und nach dem gemeinsamen Abendessen ab 20.00 Uhr noch eine Fallstudienbearbeitung oder eine Team-Building-Maßnahme anzuschließen. Zusätzlich hatten die Teilnehmer am dritten Trainingstag eine Klausur abzulegen, zu der ausreichend Vorbereitungszeit notwendig war. Das Resultat waren überforderte, unzufriedene und übermüdete Teilnehmer, die aufgrund dieser Stresssituation bei weitem nicht alle vermittelten Inhalte aufnehmen und verarbeiten konnten.

\section{Empfehlung}

Unternehmen müssen sich bewusst sein, dass die Teilnehmer aus der Praxis kommen und Schul- und Lernsituationen nicht mehr gewohnt sind. Weniger ist hier mehr: Wirksames Training beruht auf Priorisierung und pragmatischen Akzenten anstatt auf Vollständigkeit abzielenden Ausbildungskonzepten. Für den Trainingserfolg ist es demnach förderlicher, wenige, für das Tagesgeschäft relevante Inhalte zu behandeln und diese dafür in der Tiefe zu diskutieren und auf aktuelle Problemstellungen im Rahmen von Workshops anzuwenden. Darüber hinaus ist darauf zu achten, den Pausen und der Reflexion des Gelernten genügend Raum zu geben. Weiter gilt es zu berücksichtigen, dass sich die Kombination aus ambitionierten Trainingszielen und geringen Mitteln kontraproduktiv auswirkt. 
Abb. 2 Handlungsempfehlungen für den professionellen Umgang mit Inhouse-Weiterbildungsprogrammen

1. Tragen Sie unterschiedlichen Ausbildungshintergründen und Leistungsniveaus durch die Auswahl geeigneter Lehr- und Lernmethoden Rechnung.

2. Stellen Sie sicher, dass Ihre Inhouse-Weiterbildungsprogramme akademisches Wissen so vermitteln, dass es im Tagesgeschäft anwendbar und umsetzbar ist.

3. Gewährleisten Sie zur Entwicklung ganzheitlicher und nachhaltiger Weiterbildungsprogramme eine enge Zusammenarbeit zwischen Fachseite, Personalbereich und externem Partner.

4. Machen Sie die Teilnahme an RfPs für externe Anbieter so einfach wie möglich und suchen Sie frühzeitig aktiv den persönlichen Kontakt.

5. Seien Sie sich bewusst, dass sich maßgeschneiderte Weiterbildungsprogramme nur durch Ihre eigene aktive Mitarbeit während des gesamten Projekts realisieren lassen.

6. Die gewählte Weiterbildungsmethode ist nicht entscheidend. Machen Sie sich zunächst Ihre unternehmensspezifischen Probleme bewusst und wählen Sie anschließend externe Partner und Weiterbildungsformate entsprechend aus.

7. Weniger Trainingsinhalte sind oft mehr. Konzentrieren Sie sich bei der Konzeption von Trainingsinhalten auf wenige, aber relevante Aspekte, die Sie in der Tiefe diskutieren. Achten Sie auch auf ausreichend Pausen, um eine Überlastung der Teilnehmer zu vermeiden.

8. Setzen Sie nicht auf starke Einzelpersönlichkeiten oder «Startrainer», sondern vielmehr auf Partner, die in der Lage sind, den Gesamtprozess von Anfang bis Ende aus einer Hand zu begleiten und auf Ihre Bedürfnisse einzugehen.

9. Evaluieren Sie vor Projektstart die individuellen Fähigkeiten Ihrer Marketeers. Fokussieren Sie sich auf die Stärkung individueller Stärken und achten Sie darauf, dass der Teilnehmerkreis über ein einheitliches Leistungsniveau und ein gemeinsames Grundverständnis im Marketing verfügt.

10. Entwickeln Sie zuerst das Basiswissen Ihrer Marketingmitarbeiter. Stellen Sie, bevor Sie in Spezialthemen einsteigen, sicher, dass diese ausreichend Praxisrelevanz haben.

11. Seien Sie sich der Grenzen von klassischen Trainings bewusst und decken Sie wenn nötig den Transfer des Erlernten in die Praxis mit individuellen Coaching- oder Transfermaßnahmen ab.

12. Achten Sie bei der internationalen Multiplikation von Weiterbildungsprogrammen auf kulturelle Unterschiede und passen Sie Ihre Lehr- und Lernmethodik dementsprechend an.

\section{Mythos 8 - Trainerpersönlichkeit als Erfolgsgarant}

\section{Realität}

Häufig kaufen Unternehmen für viel Geld sogenannte „StarTrainer" ein oder schicken ihre Mitarbeiter zu selbsternannten oder „gehypten“ Gurus, in der Hoffnung, dass diese alle bestehenden Probleme lösen. Hingegen ziehen Star-Trainer und Gurus ihren Erfolg aus der Multiplikation ihres standardisierten Ansatzes und haben an einer unternehmensspezifischen Ausrichtung ihrer Programme kein Interesse. Des Weiteren stellen akademische Titel oder lange Publikationslisten allein noch keinen Qualitätsindikator dar. Ausgezeichnete Forschungskompetenzen sind nicht gleichbedeutend mit herausragenden Trainerfähigkeiten und Praxiserfahrung. Unternehmen wünschen sich Weiterbildungspartner mit starker Praxiskompetenz, dem Willen, auf ihre spezifischen Bedürfnisse einzugehen und der Bereitschaft, den gesamten Weiterbildungsprozess von der Akquisition, über die Konzeption bis hin zur Durchführung im Sinne eines integrierten Ansatzes aus einer Hand anzubieten.

\section{Empfehlung}

Das Beispiel eines großen deutschen Chemiekonzerns zeigt, dass Unternehmen bei der Auswahl des Weiterbildungsanbieters weniger auf starke Namen oder Einzelpersönlichkeiten achten, sondern vielmehr bestrebt sein sollten, eine wirkliche, auf persönlichen Beziehungen beruhende Partnerschaft mit dem Weiterbildungspartner einzugehen. Aus diesem Grund gilt es auf Seiten des externen Partners ein kleines Projektteam zu definieren, das in Personalunion den gesamten Prozess konzeptionell, administrativ und inhaltlich abdeckt und begleitet. Die Trainer, die das Training durchführen, sind bereits in die Akquisition und die Konzeptionsphase aktiv einzubinden, damit das gesamte Programm wirklich maßgeschneidert ist und aus einem Guss kommt. Allgemein braucht es einen Weiterbildungspartner, der sich auf das Unternehmen einlässt und sich für die Entwicklung des Unternehmens engagiert. Außerdem mag das Unterhalten der Teilnehmer im Sinne von „Infotainment“ durchaus ein wichtiger Teil von Weiterbildung sein. Sie sollte jedoch den inhaltlichen Kern einer wirksamen Weiterbildung lediglich flankieren.

\section{Mythos 9 - Trainings machen alle besser}

\section{Realität}

Derzeit sind „Corporate Academy“-Konzepte im Trend. Mit der Absicht, einen großen Teilnehmerkreis in der Breite besser zu machen, soll Marketeers im Sinne eines "Ölfilmwissens“ ein breites Spektrum an Marketingmethoden und -tools vermittelt werden. 
Dabei entsteht jedoch das sogenannte „Zehnkämpfersyndrom“ im Marketing. Es bringt durchschnittlich ausgebildete Generalisten hervor, jedoch keine Top-Performer in Einzeldisziplinen mit der Fähigkeit zur effizienten Zusammenarbeit. Hinzu kommt, dass sich der Teilnehmerkreis oft aus Personen unterschiedlicher Leistungsund Kompetenzniveaus zusammensetzt. Das führt bei einem Teil zur Überforderung, bei einem anderen zur Unterforderung und damit teilweise zu destruktivem Verhalten.

\section{Empfehlung}

Aufgrund der unterschiedlichen Leistungsniveaus der Teilnehmer sind Academy-Konzepte zu überdenken. Sicherlich braucht es gemeinsame Basismodule, in denen die Grundlagen und ein gemeinsames Marketingverständnis geschaffen werden. Jedoch müssen sich daran individuelle Vertiefungsmodule anschließen, welche nicht dazu da sind, individuelle Defizite auszugleichen, sondern vielmehr Stärken zu stärken. Hierfür bedarf es im Vorfeld eines Trainings eines Eingangstests oder eines individuellen SkillAssessments. Einen gangbaren Weg für ein solches Skill-Assessment zeigt ein deutscher Pharmazulieferer auf, dessen Personalabteilung gemeinsam mit dem Weiterbildungspartner ein zweitägiges Development Center (DC) aufgesetzt und durchgeführt hat. Erst auf Basis der DC-Ergebnisse wurden dann die konkreten Trainingsinhalte definiert, die Trainingsgruppen gebildet und die Lehrmethodik an deren Leistungsniveaus angepasst.

\section{Mythos 10 - Marketeers beherrschen ihr Handwerk \\ Realität}

Unter der Annahme, dass ihre Marketeers die Grundlagen des Marketing beherrschen, wollen Unternehmen diesen im Rahmen von Weiterbildungsprogrammen die neuesten Erkenntnisse sowie Spezialthemen im Marketing vermitteln lassen. Jedoch haben Marketeers insbesondere im Business-to-Business-Marketing häufig eine fachfremde Ausbildung, weshalb es meist an grundlegender Marketingkompetenz fehlt. Beispielsweise war es dem Marketingleiter eines deutschen Dienstleistungsunternehmens ein großes Anliegen, seinen Mitarbeitern die aktuellen Erkenntnisse im Bereich Social Media zu vermitteln. Bei der Zusammenarbeit stellte sich jedoch rasch heraus, dass die Teilnehmer nicht einmal in der Lage waren, grundlegende Marktanalyseinstrumente, wie zum Beispiel „Porter’s 5 Forces“ oder „SWOT“ richtig anzuwenden.

\section{Empfehlung}

Für Unternehmen gilt es, sich des tatsächlichen Kompetenzstands der Marketingmitarbeiter bewusst zu werden. Dieser wird sehr häufig überschätzt. In vielen Fällen geht es zunächst einmal darum, die Teilnehmer in den Grundlagen fit zu machen, bevor man in Spezialgebiete einsteigt, die für das eigentliche Tagesgeschäft nur marginale Relevanz haben.

\section{Mythos 11 -}

Klassische Trainings lösen operative Probleme

Realität

Marketeers besuchen Weiterbildungsveranstaltungen häufig mit der Erwartung, dort eine direkt umsetzbare Lösung für eine konkrete Problemstellung aus ihrem Tagesgeschäft zu erhalten. Trainingsmaßnahmen können dies aber nicht leisten. Sie sind vielmehr Impulsgeber, die Teilnehmer anregen, neue Perspektiven einzunehmen und von Best Practices zu lernen. Teilnehmer werden

\section{xelos.net business}

\section{Das Multitalent: Intranet 2.0-Software für Ihr Unternehmen!}

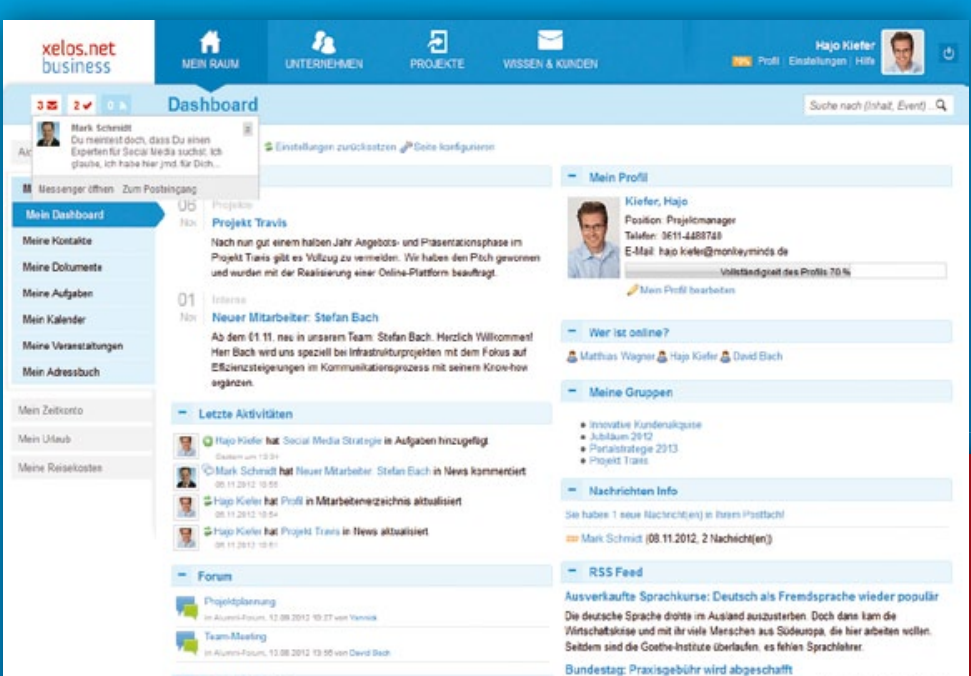

Werden Sie effizienter: Unterstützen Sie Ihr Team bei der Zusammenarbeit in Projekten und behalten Sie den Überblick über alle Aufgaben!

$\rightarrow$ Social-Activity-Stream NEU!

$\rightarrow$ intelligente Aufgabenverwaltung NEU!

$\rightarrow$ virtuelle Projekträume

W\&M-Leser erhalten $15 \%$ Preisnachlass auf die Lizenzkosten bis zum 31.03.2013!
Testen Sie xelos.net business 30 Tage kostenlos: www.xelos.net/w-m 
befähigt, nach dem Training für ihr Tagesgeschäft relevante Inhalte zu selektionieren und selbstständig Lösungen zu entwickeln. Gerade beim Transfer des Erlernten in die Praxis haben die Teilnehmer jedoch meistens die größten Probleme und benötigen individuelle Hilfestellung.

\section{Empfehlung}

Unternehmen müssen sich beim Einkauf von Weiterbildungsmaßnahmen bewusst sein, was Trainings wirklich leisten können und wo diese an Grenzen stoßen. Diese Einsicht ist auch den Teilnehmern zu kommunizieren, so dass diese nicht mit falschen Erwartungen in ein Training gehen. Darüber hinaus ist es zielführend, nach dem Training Zeit, Raum und Ressourcen für individuelle Coaching- und Transfermaßnahmen zur Verfügung zu stellen (Belz 1981). Diese können dort ansetzen, wo das Training aufhört, nämlich bei der Umsetzung des Gelernten im Tagesgeschäft.

\section{Mythos 12 - Trainings sind global multiplizierbar \\ Realität}

Nachdem eine Inhouse-Weiterbildungsmaßnahme einmal konzipiert ist, streben zahlreiche Unternehmen danach, diese so oft wie möglich international zu multiplizieren, um die Konzeptionskosten auf möglichst viele Köpfe verteilen zu können. Dabei werden

\section{Die Autoren}

Prof. Dr. Christian Belz

Direktor des Instituts für Marketing der Universität St. Gallen und Ordinarius für Betriebswirtschaft mit besonderer Berücksichtigung des Marketing an der Universität St. Gallen. Trainer in der Management-Weiterbildung

E-Mail: christian.belz@unisg.ch

\section{Dr. Michael Betz}

Leiter des Kompetenzzentrums „Inhouse and Customised Programmes" am Institut für Marketing der Universität St. Gallen. Trainer, Berater und Lehrbeauftragter für Marketing an der Universität St. Gallen

E-Mail: michael.betz@unisg.ch

\section{Philine Werner}

aber kulturelle Unterschiede in Lehr- und Lernmethoden außer Acht gelassen: So bevorzugen Seminarteilnehmer aus dem USamerikanischen Raum die Bearbeitung von abstrakten Case Studies, während Europäer lieber an realen Fragestellungen aus dem Tagesgeschäft arbeiten. In Indien werden im Rahmen von TeamBuilding-Maßnahmen nicht selten gemeinsam Tänze mit viel Körperkontakt einstudiert, während in westlichen Kulturkreisen ein formelles Gemeinschafts-Dinner oftmals schon das Höchstmaß an Annäherung darstellt.

\section{Empfehlung}

Für einen internationalen Roll-out von Weiterbildungsprogrammen ist ein „One-size-fits-all“-Ansatz ungeeignet. Vielmehr gilt es, während der Konzeption kulturspezifische Anpassungen hinsichtlich der Lern- und Lehrmethodik vorzunehmen, um die Wirksamkeit des Trainings sicherzustellen. Darüber hinaus sollten Unternehmen darauf achten, bei der Zusammensetzung des Teilnehmerkreises nicht zu viele Kulturen miteinander zu vermischen, da sonst auch hier das Trainingspotenzial nicht voll ausgeschöpft werden kann. Ausnahmen bilden Weiterbildungen, bei denen interkulturelle Kompetenz, Akzeptanz und Kommunikation im Mittelpunkt stehen.

\section{Fazit}

Vor dem Hintergrund der wachsenden Anforderungen an Marketingfach- und Führungskräfte sind Inhouse-Weiterbildungen für die Personalentwicklung von Marketeers bedeutend. Zusätzlich beanspruchen sie eine Vielzahl an monetären, zeitlichen und personellen Ressourcen, weshalb eine professionelle Auseinandersetzung mit der Thematik auf Unternehmensseite notwendig ist. Der vorliegende Beitrag zeigt in Form von ersten konkreten Handlungsanweisungen und Denkanstößen (siehe Abbildung 2) auf, wie Unternehmen bestehende Mythen entlarven und stattdessen professionell mit dem Thema Inhouse-Weiterbildung im Marketing umgehen können.

Erfolgreiche Weiterbildung in Marketing und Vertrieb setzt anspruchsvolle Strategien um, entwickelt die Potenziale der Mitarbeitenden und setzt damit Kräfte frei.

\section{Literaturverzeichnis}

Belz, Ch. (1981): Förderung des Lerntransfers in der überbetrieblichen Weiterbildung von Marketing-Führungskräften, St. Gallen. Auditorium.

DeSmet, A./McGurk, M./Schwartz, E. (2010): Getting more from your training programs, in: McKinsey Quarterly, 4.

Gök, O./Hacioglu, G. (2010): The organizational roles of marketing and marketing managers, in: Marketing Intelligence \& Planning, 28, 3, pp. 291-309.

Löhmer, A. (2012): Challenging the „Tailor“, in: The European Business Review, 2, S. 20-22.

Wyner, G. A. (2008): Marketing evolution, in: Marketing Management, 17, 2, pp. 8-9. 


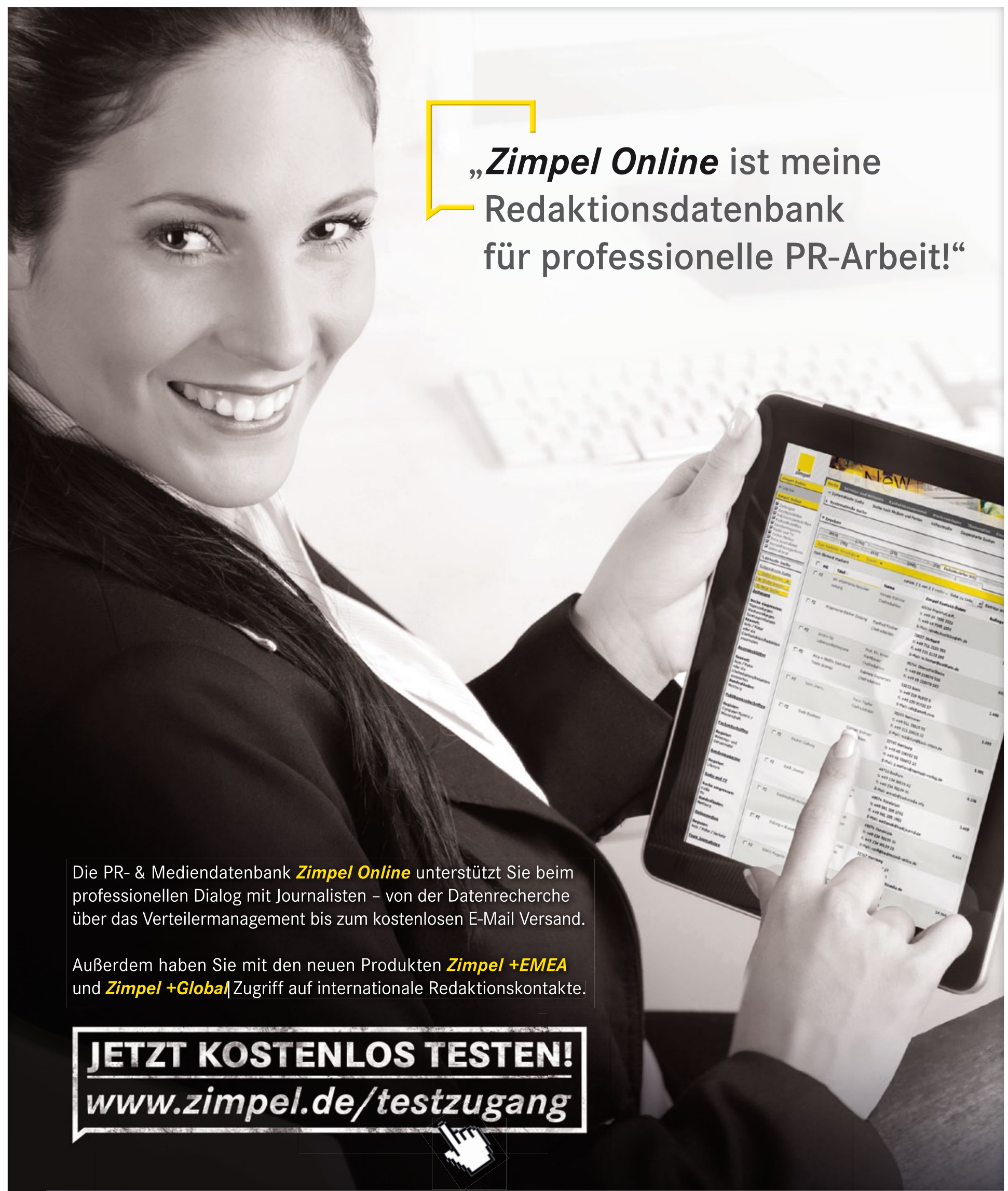

$\square$ Weitere Angebote und Informationen zu unseren Produkten finden Sie auf:

\section{www.zimpel.de}

